\section{ARE YOU PLANNING A POSSIBLE EXIT STRATEGY?}

The dental market in Scotland has experienced a significant rise in the value of NHS, fully private and mixed practices in the last 12 months with sales now regularly exceeding $100 \%$ of turnover.

Christie \& Co arrange an average of 12 viewings on every practice coming to the market for sale and $80 \%$ of what they sell goes for above the asking price, showing the appetite amongst buyers and the current imbalance of supply and demand. Generally all buyer profiles - corporates, multiple operators, independents and first time buyers - are active with each bringing certain attributes to the market.

In recognition of the different needs and demands of these buyer types, Christie \&t Co offers a bespoke service tailored specifically for you the seller! Unlike many agents who agree to take fees from both buyer and seller, Christie \&t Co views this as a conflict of interest. Taking a confidential 'whole market approach' to selling they have the expertise to deliver your objectives in a fair market place.

In 2016 you may well be planning a possible exit strategy. If you are considering a sale within the next five years then it is important to begin the process now. To some this may seem too far down the line but it is imperative to do so should you wish to maximise the result.

To discuss how Christie \& Co might help you achieve your future plans in Scotland contact Paul Graham on 01315243416.

\title{
APP TRACKS PROGRESS WITH ALIGNER TOOTH STRAIGHTENING
}

Align Technology, makers of the clear aligner teeth straightening system which has treated over 3 million patients worldwide, has launched an app which is available for free to download. The app - 'My Invisalign Smile' - works as a companion to support anyone undergoing Invisalign teeth straightening, and features a number of functions which make keeping track of their orthodontic treatment easier, as well as interesting and fun.

Invisalign aligners are changed in two weekly intervals and the app's image function includes a built-in guide to allow users to capture consistently positioned camera shots of their face, their smile and their teeth as they transition towards their final, corrected position. These shots build up into a gallery which also allows the person undergoing treatment to compare their changing teeth, side-by-side to see exactly how their new smile is taking shape.

It is also possible to create powerful time-lapse videos which display the user's teeth transformation story as it unfolds, capturing and demonstrating just how far they have progressed as their dentition moves incrementally over the weeks.

Another function of the app captures this sense of growing anticipation by allowing users to share images and videos of their transitioning appearance with friends and family and on social media.

The app also provides those in treatment with regular tailored support, advice and reminders (including prompts to change their aligners every two weeks or attend their next dental appointment) synchronised with their individual treatment plan, so that they stay on the right track throughout their treatment.

For more information visit http://www.invisalign.co.uk/ myinvisalignsmile.

\section{NEW LEARNING FOR ASPIRATIONAL} YOUNG DENTISTS

Aimed at providing a practical springboard from which newly-qualified professionals can launch into a career of excellence, Step Education has been specifically designed to fulfil the needs of young dentist in the first five years of general practice.

By offering an eclectic range of pertinent topics, from personal development, to clinical skills and business management, Step Education can prepare you for a long and successful career in the competitive dental profession.

With the chance to learn from some of the UK's best speakers and professional mentors, including Anthony Summerwill, James Hamill, Michael Waplington, Robert Oretti, David Wilson, Martin Evans, Louis Mackenzie, Myke Woollett, Allan Suggett and Chris Barrow, the expertise and experience on offer is unsurpassable.

Make sure you get the best start to your career: develop your skills, learn how to be successful and network with your peers in the most important time of your professional life. Contact the Step Education team today to find out more.

To learn more about Step Education visit www.stepeducational.com, email booking@stepeducational. com or call 08001303573.

\section{TOOTH POLISH WITH COCONUT SHELL CHARCOAL}

The Diamond Whites Black Edition Tooth Polish is a $100 \%$ natural tooth polish made from 100\% activated coconut shell charcoal.

The Tooth Polish is designed to change the way patients whiten their teeth and also strengthen the gums, remove toxins from the mouth and absorb bacteria.

To use Diamond Whites Tooth Polish, you dip a wet toothbrush into the powder then brush your teeth in a small circular motion, rinse well and spit carefully into the sink.

Diamond Whites is a cosmetic teeth whitening company established in 2012 with a UK distributor in Hertfordshire. Its products fully comply with EU Regulations and do not release more than 0.1\% hydrogen peroxide.

The Diamond Whites Black Edition Tooth Polish is available now from http://www. diamondwhites.co.uk/ product/black-edition/ at a cost of $£ 14.99$.

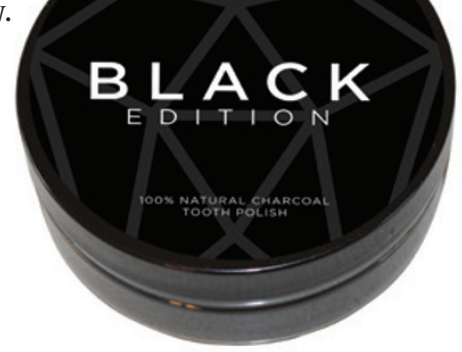

